\title{
Assistenz statt Hilfe
}

\section{Das Projekt »Lokale Verantwortungsgemeinschaften in kleinen Lebenskreisen «}

\author{
Ulrike Overkamp
}

Ulrike Overkamp ist Altenpflegerin und Diplom-Pädagogin; sie verfügt über Fachausbildungen in Gruppendynamik und Selbstorganisation (DAGG) und als Supervisorin (DGSv). Sie ist im Ev. Johanneswerk in Bielefeld tätig und Leiterin des Projektes »Lokale Verantwortungsgemeinschaften in kleinen Lebenskreisen $(\mathrm{LoVe}) \ll$. E-Mail

Ulrike.Overkamp@johanneswerk.de
Fach- und Führungskräfte aus der Sozialen Arbeit erproben in einem neuen Projekt die gleichberechtigte Zusammenarbeit mit Pflege- und Assistenzfachkräften sowie mit bürgerschaftlich engagierten Menschen. Die Idee: Selbstbestimmung und Teilhabe sind für Menschen mit Unterstützungsbedarf am besten erreichbar, wenn Angehörige, Nachbarn, bürgerschaftlich Engagierte und Fachkräfte im Wohnquartier gemeinsam Verantwortung für diese Menschen übernehmen.

Die im Netzwerk »Soziales neu gestalten (SONG) « verbundenen Akteure vertreten den Anspruch, die Herausforderungen des sozialen Wandels der kommenden Jahre und Jahrzehnte aktiv zu gestalten. Ein Gestaltungselement stellt das gemeinsame Projekt »Lokale Verantwortungsgemeinschaften in kleinen Lebenskreisen (LoVe)« dar. Die Vision des Netzwerks besteht in einem lokal-kooperativen Sozialmodell, da die Partner überzeugt sind, dass die sozialen Herausforderungen nur am Lebensort der Menschen in enger Kooperation der lokal Verantwortlichen lösbar sind.

\section{Das Projektvorhaben}

Das Qualifizierungsprojekt »Lokale Verantwortungsgemeinschaften in kleinen Lebenskreisen (LoVe) « soll Mitarbeiterinnen und Mitarbeiter zur Entwicklung und Umsetzung neuer quartiersbezogener Wohn- und Assistenzkonzepte befähigen. Es eröffnet dadurch auch neue Perspektiven für den Arbeitsmarkt. Dabei kann auf die bereits vorhandenen Konzepte des Netzwerks SONG aufgebaut werden.

Die alternde Gesellschaft ist eine der größten Herausforderungen des 21. Jahrhunderts. Aufgrund des zu erwartenden Anstiegs von Assistenzbedürftigkeit stellt sich die Frage, wie ein selbständiges und zufriedenstellendes Altern gelingen kann. Selbstbestimmung und Teilhabe sind
Werte, die nicht nur für die älter werdende Bevölkerung, sondern auch für die ebenfalls zunehmende Zahl von behinderten Menschen an Bedeutung gewinnen. Nicht zuletzt durch die UN-Konvention über die Rechte behinderter Menschen geht es darum, Voraussetzungen für die Inklusion in der Gesellschaft zu schaffen.

Der demographische Wandel führt nicht nur zur Zunahme der Zahl pflegebedürftiger Menschen, sondern auch zur Veränderung der Altersstruktur der Beschäftigten in Sozialberufen. Die Zahl langjähriger und älterer Mitarbeiterinnen und Mitarbeiter steigt. Nicht wenige von ihnen sind allerdings durch die zunehmende Rationalisierungs- und Effizienzorientierung der herkömmlichen Versorgungssysteme überfordert. Dieser Herausforderung kann nicht ausschließlich mit den Instrumenten der Frühverrentung und Altersteilzeit begegnet werden. Insbesondere für diese Personen bedeuten jedoch die angestrebten lokalen Verantwortungsgemeinschaften eine Chance zum Erhalt ihrer Motivation, Gesundheit und Beschäftigungsfähigkeit.

Die durch die neuen Assistenzmodelle gegebenen Arbeitsbedingungen sollen dazu beitragen, dass die älter werdenden und von Krankheit bedrohten Beschäftigten möglichst lange im Beruf verbleiben können. In einer Gesellschaft des langen Lebens werden zudem die Potenziale der älteren Menschen im Ruhestand neu entdeckt. Die quartiersbezogenen Wohnund Assistenzmodelle sowie das professionelle Netzwerkmanagement eröffnen diesem Personenkreis neue Möglichkeiten, sich als Teil der lokalen Verantwortungsgemeinschaften entsprechend den individuellen Möglichkeiten und Neigungen bürgerschaftlich zu engagieren. Insbesondere die Qualifizierung auf die Funktion als neutrale Moderatoren eröffnet bürgerschaftlich Engagierten eine verantwortungsvolle Engagementmöglichkeit. 
Assistenz- und Führungskräften eröffnen sich neue Formen der Arbeit; ihre Beschäftigungschancen werden auf die künftigen neuen Anforderungen hin verbessert. Sie erwerben über die handlungsorientierte Didaktik eine breite Palette von sozialen und methodischen Kompetenzen, die weit über die Anforderungen in den lokalen Verantwortungsgemeinschaften hinausgehen und somit die Beschäftigungsfähigkeit generell erhöhen. Nicht zu vernachlässigen sind die neuen das Personalentwicklungsprogramm » rückenwind « für Beschäftigte in der Sozialwirtschaft des Europäischen Sozialfonds sowie durch Eigenmittel der SONG-Träger, gehen die Beteiligten neue Wege.

Im Netzwerk haben sich die Organisationen Bremer Heimstiftung (mit Sitz in Bremen), das Evangelische Johanneswerk e.V. (Bielefeld), die Caritas Betriebs- und Trägergesellschaft Köln, die Stiftung Liebenau (Meckenbeuren), das Kuratorium

\section{"Neue Allianzen stellen sich der Verantwortung für Leben, Wobnen und Versorgung im Quartier"}

sinngebenden Engagementmöglichkeiten für aktive, lebenserfahrene Bürger (z. B. während der Familienphase oder im Seniorenalter); auch diese Personengruppe wird für ihre verantwortungsvolle Aufgabe auf der Basis des erarbeiteten Konzepts durch die SONG-Partner geschult. Die Qualifizierungen dienen somit insgesamt der Verbreitung der zukunftsfähigen Assistenzmodelle sowie der Sicherung von Kompetenzen und Beschäftigungsfähigkeit der Zielgruppen.

\section{Neue Versorgungssysteme zwischen dem ambulanten und stationären Regelsystem}

Die quartiersnahe Versorgung tritt an, das ambulante und stationäre Versorgungsangebot der Alten- und Behindertenhilfe zu ergänzen und den Wünschen weiter Teile der Bevölkerung im Kontext von Pflege- und Assistenzbedarfen zu entsprechen: Versorgungssicherheit kleinräumig, in geteilter Verantwortung zwischen professionellen Kräften und der Laienpflege $\mathrm{zu}$ realisieren ist ihr Anspruch.

Erworbene Kompetenzen wertzuschätzen und gleichzeitig neue Wege zu gehen, um Wohnformen zu realisieren, die den Bedürfnissen und Wünschen der meisten Menschen entsprechen, ist das Ziel des Qualifizierungsprojekts. Finanziert durch
Deutscher Altershilfe (Köln), die Bank für Sozialwirtschaft (Köln) und als Kooperationspartner die Stiftung Bürgermut und für das Projekt LoVe die Bundesakademie für Kirche und Diakonie in Berlin zusammengeschlossen. Sie realisieren schon jetzt Dienstleistungsstrukturen, die entsprechend dem Welfare-Mix-Ansatz »lokale Verantwortungsgemeinschaften in kleinen Lebenskreisen « ermöglichen. Sie vertreten den Anspruch, die Herausforderungen des sozialen Wandels der kommenden Jahre und Jahrzehnte durch unterschiedliche Maßnahmen zu gestalten.

Innovative quartiersbezogene Assistenzmodelle im Sozialraum sind ein neuer Ansatz gegenüber den bisherigen Tätigkeitsschwerpunkten der Träger in der Alten- und Behindertenhilfe. Der sozioökonomische Mehrwert der Modelle liegt in der höheren Lebensqualität, Selbstbestimmung und Teilhabe insbesondere älterer und behinderter Menschen sowie in den zukunftssicheren, hilfebedarf- und kostenbegrenzenden Assistenzkonzepten. Eine wissenschaftliche Untersuchung hat für die von den SONG-Partnern bisher realisierten quartiersbezogenen Wohnund Assistenzmodelle im Vergleich zu den herkömmlichen Wohn- und Betreuungsformen insbesondere folgende Vorteile analysiert:

- stärker ausgeprägtes soziales $\mathrm{Zu}$ sammenleben und verbesserte Kommunikation
- bessere Gesundheitsentwicklung und geringerer Hilfebedarf der Bewohnerinnen und Bewohner

- intensivierter Austausch mit Nachbarn und erhöhtes Engagement der Quartiersbewohnerinnen und Quartiersbewohner

- mehr Angebote und stärkere Inanspruchnahme von Nachbarschaftshilfe

- messbare Einspareffekte für Bürgerinnen und Bürger sowie öffentliche Kostenträger

- positive Integrationseffekte für weitere Personengruppen

(vgl. Netzwerk: Soziales neu gestalten [Hrsg.]: Zukunft Quartier - Lebensräume zum Älterwerden, Band 3: Soziale Wirkung und »Social Return«. Eine sozioökonomische Mehrwertanalyse gemeinschaftlicher Wohnprojekte. Gütersloh 2009)

Der bisherige Einsatz und die Qualifizierung der Mitarbeiterinnen und Mitarbeiter sind auf traditionelle Pflege- und Betreuungssettings ausgerichtet. Das systematische Qualifizierungsangebot zur Realisierung der neuen Assistenzmodelle mit den genannten drei Zielgruppen ist daher eine neue Form der Personalentwicklung. Orientiert an den neuen Modellen werden Kompetenzprofile entwickelt und eine empirisch abgesicherte Potenzialanalyse durchgeführt. Auf diese Weise wird sichergestellt, dass die Qualifizierung die Passung von beruflichen Anforderungen und persönlichen Kompetenzen verbessert.

Das bisher einmalige Kooperationsprojekt LoVe trägt mit drei verschiedenen Qualifizierungsangeboten zur Personalentwicklung zur Weiterentwicklung von Versorgungssystemen im lokalen Nahraum bei. Es qualifiziert insgesamt 110 Mitarbeitende der Netzwerkpartner, die sich in den nächsten Jahren auf drei Ebenen weiterbilden werden:

- einer Managementebene mit der Aufgabe, quartiersorientierte Angebote, Dienstleistungen und Netzwerke zu entwickeln und zu steuern (30 Führungskräfte. Qualifizierungsmodul »Lokales Dienstleistungs- und Netzwerkmanagement $«$ )

- einer Ebene der Assistentinnen und Assistenten, die Pflege- und Assistenzleistungen im Quartier erbringen (40 Fachkräfte der Alten- und Behindertenhilfe. Qualifizierungsmodul »Sozialraumorientierte Assistenz «) sowie 
- einer Ebene für zivilgesellschaftlich engagierte Bürgerinnen und Bürger als Mediatorinnen und Mediatoren und Interessenvertretungen aller im Quartier lebenden Bewohnerinnen und Bewohner (40 bürgerschaftlich engagierte Bürgerinnen und Bürger aus den Quartieren. Qualifizierungsmodul »Neutrale Bürgermoderation «).

Für die zukünftigen Mitarbeiterinnen und Mitarbeiter in den Quartiersmodellen stellen sich eine Reihe von herausfordernden Situationen. Ihr Berufsalltag ist geprägt sowohl von operativen Aufgaben im Dienstleistungsbereich als auch von weitergehenden strukturellen und strategischen Anforderung durch Vernetzung und Implementierung von Welfare-MixStrukturen. Dies unter der Voraussetzung, dass sich die Ansprüche der Kunden und Menschen im Quartier diversifizieren und durch abnehmendes Hilfepotenzial neu ordnen müssen.

Die multiprofessionell ausgestalteten Teams in den Versorgungseinheiten in den Quartiersprojekten setzen dabei weniger auf Konkurrenz der unterschiedlichen Berufsgruppen als vielmehr auf deren Kooperation. Diese ist unerlässlich, will man die Versorgung von Menschen im Quartier kleinräumig gestalten und realisierbar umsetzen. Unter der Voraussetzung, dass sich hier neue Allianzen bilden, die eine gemeinsame Verantwortung für das Leben, Wohnen und die Versorgungssicherheit implementieren sind alle Berufsgruppen im Bereich der Sozialen Daseinsvorsorge angesprochen.

Auf diesem Hintergrund werden sich die Qualifizierungsmaßnahmen durch eine modulare Struktur kennzeichnen. In insgesamt sieben Modulen, die sich als Pflicht-, Wahlpflicht und Wahlmodule realisieren, werden in verschiedenen Kompetenzbereichen Wissenszuwächse erwartet. Als wesentliche Kompetenzbereiche sind durch Interviews und teilnehmende Beobachtung verschiedener Zielgruppen Kompetenzbereiche identifiziert worden:

- Wohnungsbau (Barrierefreiheit und Barrierearmut) und Wohnkonzepte für verschiedene Zielgruppen

- rechtliche Fragestellungen in den Bereichen SGB V, IX, XI, XII

- betriebswirtschaftliche Kenntnisse

- Fachkompetenzen in Marketing und Öffentlichkeitsarbeit

- Qualitätsmanagement
- sozial- und pflegewissenschaftliches Know-how sowie

- Kenntnisse zur regionalen Infrastruktur Daneben tragen herausfordernde Situationen für die Mitarbeiterinnen und Mitarbeiter vor Ort zu Kompetenzentwicklungen im Bereich der personalen und sozialen Kompetenzen bei. Beispielhaft sind nachfolgende Situationen genannt worden:

- Überalterung der Mieterinnen und Mieter

- die Versorgung von Menschen mit Demenz

- Vernetzung und Gründung sozialer Arbeitskreise von Profis und Laien

- mangelnde strukturelle Absicherung, beispielsweise durch Kommunen

- unzureichende Ausstattung mit Sachmittel wie EDV
- Einbindung von Menschen mit Behinderungen

- Realisierung des Hilfe-Mixes

- schlechte räumliche Ausstattung

- extreme Verhaltensauffälligkeiten bestimmter Zielgruppen

Die komplexen Anforderungen an eine kleinräumige quartiersnahe Versorgung verschiedener Zielgruppen mit Hilfeund Assistenzbedarfen sind durch eine Berufsgruppe allein nicht zu bewerkstelligen.

Die Erfahrungen zeigt, dass Kooperation und Zusammenarbeit in den Teams nicht reibungslos und konfliktfrei verlaufen, jedoch für den Erfolg solcher Modelle unersetzlich ist. Aus unserer Erfahrung gelingt die Zusammenarbeit dann, wenn eine entsprechende Personalentwicklung diese stützt.

\section{Das »Netzwerk: Soziales neu gestalten (SONG)《}

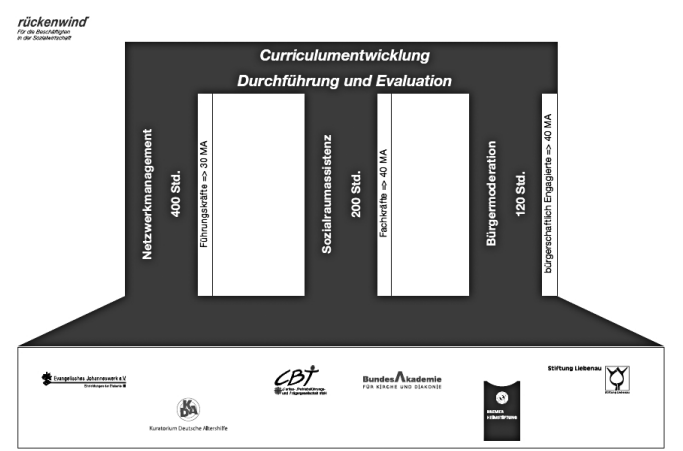

ist ein Zusammenschluss von verschiedenen Akteuren in der Sozialwirtschaft. Ihr gemeinsames Fundament ist ihr Engagement für das Gemeinwohl und der Wille die Zukunft aktiv und gemeinsam $\mathrm{zu}$ gestalten. Die Aktivitäten in diesem Netzwerk werden auf die Themen fokussiert, die einen entscheidenden Einfluss auf die $\mathrm{Zu}-$ kunftsfähigkeit der Gesellschaft haben. Dabei spielen die Themenfelder Alten-, Behinderten- und Jugendhilfe eine große Rolle.

Durch die Professionalisierung und die teilweise Überregulierung im Sozialbereich sind die primären Hilfesysteme wie Familien und Nachbarschaftshilfen immer mehr aus dem Blickfeld geraten. Alle Netzwerkpartner teilen die Überzeugung, dass soziale Leistungen für die Zukunft einerseits dem Wunsch der Menschen nach Eigenverantwortung und Selbstbestimmung zu entsprechen haben und andererseits nachhaltig zu sichern sind. Daher müssen sich soziale Leistungen verstärkt an Solidarität, Subsidiarität, sozialräumlichen Lösungen und bürgerschaftliche Eigenverantwortung orientieren. Eine solche Haltung stiftet letztendlich durch die Möglichkeit der Teilhabe auch bei den Menschen mehr Sinn als eine auf Versorgung und Konsum sozialstaatlicher Leistungen orientierten Einstellung. Vor diesem Hintergrund suchen alle operativen Netzwerkpartner nach ergänzenden Angebotsstrukturen zur stationären Versorgung.

Ein Projekt des Netzwerkes ist »Lokale Verantwortungsgemeinschaften in kleinen Lebenskreisen (LoVe) «. Das vom Evangelischen Johanneswerk für SONG maßgeblich getragene Innovationsprojekt will gemeinsam mit der Fachhochschule Münster und der Bundesakademie für Kirche und Diakonie entsprechende Curricula entwickeln. Zunächst werden projektinterne Kursen konzipiert, die zum lokalen Dienstleistungs- und Netzwerkmanagement, sozialraumorientierten Assistenz und neutralen Bürgermoderation qualifizieren sollen.

Internet http://www.netzwerk-song.de 